\title{
Coulisses
}

Revue de théâtre

19 | Hiver 1999

Varia

\section{Lip : les horlogers remettent les pendules à l'heure}

\section{Cyril Brossard}

\section{OpenEdition}

Journals

Édition électronique

URL : https://journals.openedition.org/coulisses/538

DOI : 10.4000/coulisses.5381

ISSN : 2546-9460

\section{Éditeur}

Presses universitaires de Franche-Comté

\section{Édition imprimée}

Date de publication : 1 janvier 1999

Pagination : 14-16

ISBN : 2-913322-09-3

ISSN : $1150-594 \mathrm{X}$

Référence électronique

Cyril Brossard, « Lip : les horlogers remettent les pendules à l'heure », Coulisses [En ligne], 19| Hiver 1999, mis en ligne le 18 octobre 2019, consulté le 04 janvier 2022. URL : http://

journals.openedition.org/coulisses/5381; DOI : https://doi.org/10.4000/coulisses.5381

Ce document a été généré automatiquement le 4 janvier 2022

Coulisses 


\section{Lip : les horlogers remettent les pendules à l'heure}

\section{Cyril Brossard}

1 Tenter une histoire de Lip aujourd'hui n'est pas sans déchaîner les passions. Celle-ci se trouvera confrontée aux réactions de ceux qui ont été les acteurs d'une telle lutte. Ce simple fait montre la prégnance du mouvement dans la mémoire collective. Plusieurs témoins parlent d'ailleurs de la trop forte exploitation de cette histoire par des chercheurs ou écrivains en tous genres, qui s'approprient à leur profit un tel

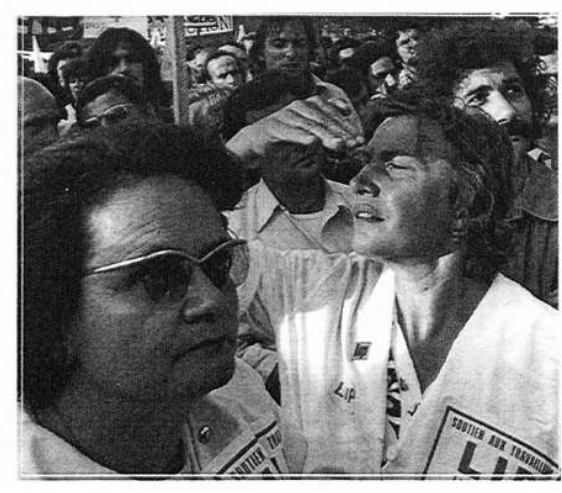
événement, et éditent des ouvrages parfois éloignés de la réalité en utilisant l'impact du nom Lip. Certes ce reproche peut sembler justifier aux yeux des acteurs de ce qui s'est passé en 1973, mais il convient de nuancer. En effet, les employés Lip ont su utiliser les médias, la presse pour populariser leurs actions. Ce faisant, une partie de leur histoire leur échappait et tombait dans le « domaine public ». Il est clair qu'en attirant l'opinion publique et intellectuelle sur les problèmes économiques et sociaux qui se posaient à Palente, les «Lip » perdaient l'exclusivité de leurs actions.

Quoi qu'il en soit, il ne nous appartient pas dans cet article de faire une histoire de Lip mais de montrer les formes de luttes et de solidarité qui ont animé ceux qui ont ébranlé le calme de l'été 1973. Enfin, il convient d'être prudent en évoquant les acteurs puisque si l'affaire s'est déroulée à Palente, tous ne résidaient pas dans le quartier, ni même dans la ville. Le mouvement Lip ne se résume donc pas à la rébellion et à la solidarité des Palentais. 


\section{Ce qu'est Lip} industriel de Besançon. atelier à Besançon en 1867. Il faut attendre 1959 et la nécessité de s'agrandir pour voir s'installer cette entreprise sur un terrain de quarante hectares à Palente-Village. Une usine modèle avec de très larges salles est prévue. Ce sont donc plus de 1450 ingénieurs, mécaniciens, horlogers spécialistes et des laboratoires de pointe qui arrivent dans le quartier.

5 La réussite industrielle semble être complète; pourtant, au printemps de 1973, le groupe est en difficultés financières. Le président, M. Saintesprit, démissionne du comité d'administration et deux administrateurs provisoires sont désignés par le tribunal de commerce.

6 L'enjeu est clair : pour redresser l'entreprise, il faut négocier avec d'autres sociétés. Que s'est-il passé entre temps?

\section{Les ferments de la crise}

7 Lip n'était pas seulement spécialisée dans le domaine de l'horlogerie, elle se battait aussi sur les marchés de l'équipement civil et militaire, la mécanique, l'usinage et les machines outils. Son équipement industriel et sa main d'œuvre ont donc été utilisés pour des produits de précision différents de l'horlogerie, laquelle fut un temps délaissée au moment où arrivait la concurrence étrangère.

Les difficultés financières de l'industrie seraient venues également de la personnalité originale de Fred Lip. Les sursalaires, le sureffectif et la surqualification seraient une autre cause des déficits du groupe selon les anciens directeurs de l'usine, qui ajoutent qu'il y avait $45 \%$ de personnel non productif dans l'entreprise. Fred Lip avait le tort de vouloir assurer de bonnes conditions de travail et de rémunération à ses employés au moment où le chiffre d'affaire ne le permettait plus.

C'est dans ce contexte troublé que le trust suisse Ebauches SA prend, en 1971, le contrôle du groupe Lip, et élabore un plan de licenciements et de démantèlement que les travailleurs découvrent en mars 1973 au cours du comité d'entreprise. La CGT y voit le démantèlement de Lip à long terme, sa transformation en une simple usine de montage de Ebauches SA. La CFDT rejoint cette idée.

\section{La lutte pour la survie}

Les ouvriers réagissent immédiatement, créent un comité d'action, ralentissent les cadences, organisent des commissions de luttes, mettent à l'abri un stock de 100.000 montres et font redémarrer le travail pour leur propre compte, la première paye sauvage a lieu le 3 août mais le bilan est bientôt déposé et «les forces de l'ordre font évacuer l'usine au cœur de l'été. Tandis que de longues négociations s'engagent, les Lip popularisent leur lutte et parviennent à réunir à Besançon, le 29 septembre, près de 100.000 personnes lors de la célèbre «marche Lip ». (...) Des accords sont signés à Dole

Coulisses, 19 | Hiver 1999 
le 29 janvier 1974 sur la base d'un plan présenté par Claude Neuschwander. Celui-ci devient bientôt le PDG d'une nouvelle société, la Compagnie européenne d'horlogerie, qui démarre en mars 1974. $»^{1}$

\section{Au terme d'une année de luttes, que dire du conflit Lip?}

11 C'est d'abord l'originalité de la lutte qui frappe les esprits. La production de montres est illégale, le stock qui a été pris pourrait être qualifié de vol. Les cadres de l'usine qui avaient voté la reprise du travail se rebiffent devant de telles méthodes. Dans un conflit classique, les travailleurs provoquent l'épreuve de force, le patron laisse le temps agir pour lui pour ramener les employés à leur poste. Chez Lip, la production sauvage et les 100.000 montres en stock constituent une arme et un moyen de subsister; le temps est avec eux. Est-ce une nouvelle forme de production? Le mot autogestion est lâché. Elle élimine le pouvoir de la hiérarchie et remet en cause le système capitaliste pyramidal. Les travailleurs sont capables de s'organiser eux-mêmes, de produire, de vendre. Un échange de savoir-faire se transmet entre l'ouvrier spécialisé et les agents techniques : "chacun apprend de l'autre et apprend des autres pour la lutte et pour son épanouissement personnel. $»^{2}$ Lors de la "paye sauvage ", les Lip ont donné $10 \%$ pour la caisse de solidarité avec les travailleurs en lutte dans d'autres entreprises et dans d'autres régions.

Ce sentiment d'entraide a pris une proportion internationale puisque les sidérurgistes allemands et les horlogers suisses avaient pris l'engagement d'empêcher tout transfert de la production de Lip vers leur pays respectif. Un bémol toutefois, une certaine hiérarchie dans les salaires est demeurée, ce qui constitue une limite à la solidarité. Par ailleurs, l'unité entre les syndicats, et entre les travailleurs syndiqués et les autres, ne semble pas si évidente. La CFDT était plus active que la CGT. Charles Piaget, son leader, a maintenu le mouvement malgré des divergences de vues. Les délégués faisaient tout et siégeaient en permanence, alors que les non délégués restaient inactifs, coupés des décisions.

13 La solidarité syndicale, dont on vient de voir la limite, se double d'une solidarité chrétienne dans laquelle l'Eglise a eu un rôle. L'abbé Princet, actuel prêtre de l'église St Pie X, à Palente, témoigne : "Je n'ai pas connu Lip parce que je n'étais pas ici. J'en ai entendu parler étant au centre-ville. Je n'étais pas aussi proche d'eux que le père Manche. L'abbé Manche n'a rien marqué sur Lip dans les archives paroissiales au moment des événements. Lip a fait l'unanimité en tant qu'événement important dans toute la France. Besançon, c'était Lip. En Bretagne, il y avait un panneau de parking « $\mathrm{P}$ » avec « LI » devant... en Bretagne ! Il y avait une solidarité ouvrière de Lip. Au Lux, se tenaient les Assemblées Générales, ainsi que dans les sous-sols de l'église. » Retenons que Marcel Manche n'a pas voulu ou pu conserver de témoignages ayant trait à l'affaire. L'abbé serait intervenu plus activement le lendemain du 14 août, jour de la charge des gendarmes mobiles; il fut «au cœur de la lutte de tous les jours, en y apportant sa contribution matérielle, en multipliant ses efforts sur le terrain, en intervenant aussi là où l'affrontement s'était déplacé, dans la coulisse des tentatives de négociations (...) Le plus spectaculaire et le plus connu, c'est naturellement l'appui logistique apporté aux Lip par celui qui était devenu « le curé des Lip ». (...) La cure de St Pie X est devenue une sorte de siège social pour les Lip. ${ }^{3}$ L'abbé Manche avait choisi 
son camp. Il soutenait les contestataires mais apportait une caution morale au conflit. Homme d'église, il s'est posé contre un certain ordre laïc et a entraîné ses supérieurs religieux dans ses choix. Le père de la paroisse de Palente a peut-être plus agi en homme de proximité et de terrain selon sa conscience, qu'en clerc.

Pourtant, en impliquant l'Eglise dans cette lutte, les risques étaient grands que l'institution tout entière se fît critiquer. En octobre 1973, le rédacteur de la revue Etudes sociales et syndicales, après avoir qualifié l'affaire Lip d'« histoire de curé », s'en prenait à l'archevêque de Besançon et à l'ingérence «de l'irrationnel dans les problèmes sociaux!»

Il semble qu'il y ait eu plusieurs clergés, plusieurs façons de vivre l'Evangile dans le conflit : pour l'Association Catholique Ouvrière, Lip était un exemple frappant d'une économie basée uniquement sur l'intérêt de quelques-uns au détriment du bien général d'une localité.

L'archevêque, lui, parlait un langage destiné essentiellement aux patrons et aux classes dominantes. Jean Raguenes, dominicain, ouvrier spécialisé chez Lip, se spécialisait dans les discours aux ouvrières pour "mobiliser et entraîner les hommes par leur intermédiaire et pour tenir ces femmes dans l'action jusqu'au bout. »

17 L'abbé Manche, enfin, semble avoir assumé le rôle maternel de l'Eglise par sa protection, son entraide, son secours. Il a évité les actes nuisibles et a ouvert les portes de sa cure quand les travailleurs furent expulsés de l'usine.

La solidarité chrétienne existe, c'est indéniable ; mais elle semble divisée, fractionnée à l'instar des situations professionnelles que compose le personnel de l'usine. Ce fut très probablement un des points faibles du mouvement.

Les Lip ont renversé la logique industrielle. Leur aventure est devenue un idéal romantique. Deux thèmes ont été mis en valeur : la région et l'autogestion. «Continuer à vivre au pays » était une formule choc dans un monde qui commençait à contester les grandes concentrations, les ordres venus de l'étranger, les délocalisations et l'éclatement du personnel et de l'outil de travail.

20 En réalité, il y eut d'abord à Besançon un souci d'autodéfense, de survie plus que de transformation de la société. Il s'agissait de sauver l'entreprise et de rechercher un patron efficace.

21 Au-delà des frontières de Franche-Comté, les Lip sont devenus un symbole détesté par les uns, admiré par les autres. Ils ont su jouer des médias pour rallier l'opinion publique à leur cause et les faire intervenir comme second acteur. Grande affaire sur les principes, Lip n'a pourtant guère influencé les statistiques des entreprises en difficulté. Mouvement social quantitativement minoritaire dans une France de 1973 assez calme, il n'en a pas moins été un mouvement sociétal majeur dans sa force d'impact sur les Français et les pays étrangers.

L'année 1973 ne marque pourtant pas la fin de l'histoire de l'entreprise. La lutte pour la survie continue jusqu'au milieu des années 1980, ponctuée par des luttes plus ou moins violentes des employés. Le patrimoine de Lip est pourtant vendu en 1983-84. Selon les mots d'un ministre en place quatorze ans auparavant : «Lip, c'est fini !». Mais après combien d'années de luttes et de sursis gagnés! Une question reste posée : quel fut l'impact, positif ou négatif, d'un tel événement sur la volonté des entreprises à venir s'installer dans un quartier aussi « contestataire »? 


\section{NOTES}

1. Charles Piaget et al., Les Lip racontent, Stock 2, 1973.

2. Charles Piaget et al., ibid.

3. Mgr Dalloz et al., A Dieu, l'abbé, Besançon, 1988

\section{AUTEUR}

\section{CYRIL BROSSARD}

Auteur de Palente, la solidarité au quotidien, 1998, ouvrage qui retrace l'histoire du quartier, édité et disponible à la MJC de Palente. 